\title{
Coherent Compton Effect
}

\section{Citation}

Golovchenko, J. A., D. R. Kaplan, B. Kincaid, R. Levesque, A. Meixner, M. F. Robbins, and J. Felsteiner. 1981. Coherent Compton Effect. Physical Review Letters 46, no. 22: 1454-1457. doi:10.1103/physrevlett.46.1454.

\section{Published Version}

doi:10.1103/PhysRevLett.46.1454

\section{Permanent link}

http://nrs.harvard.edu/urn-3:HUL.InstRepos:29407054

\section{Terms of Use}

This article was downloaded from Harvard University's DASH repository, and is made available under the terms and conditions applicable to Other Posted Material, as set forth at http:// nrs.harvard.edu/urn-3:HUL.InstRepos:dash.current.terms-of-use\#LAA

\section{Share Your Story}

The Harvard community has made this article openly available.

Please share how this access benefits you. Submit a story.

Accessibility 
and electron-capture collision processes.

In conclusion, an initial attempt has been made to investigate the effects of strong dc electric fields on collisions of ions with Rydberg atoms. The changes in the calculated cross sections as a function of field strength are found to be quite large making experimental observations feasible. Hence, future studies of these processes should readily lead to progress in this subset of collision physics.

This work was supported by the Physics Division of the U. S. Office of Naval Research.

\footnotetext{
(a) Present address: Physics Department, University of Missouri-Rolla, Rolla, Missouri 65401.

${ }^{1}$ P. M. Koch and J. E. Bayfield, Phys. Rev. Lett. 34, 448 (1975).

${ }^{2}$ M. Burniaux, F. Brouillard, A. Jognaux, T. A. Govers, and S. Szucs, J. Phys. B 10, 2421 (1977).

${ }^{3}$ H. J. Kim and F. W. Meyer, Phys. Rev. Lett. 44 , 1047 (1980).
}

${ }^{4}$ K. B. MacAdam, D. A. Crosby, and R. Rolfes, Phys. Rev. Lett. 44, 980 (1980).

${ }^{5}$ I. C. Percival and D. Richards, Adv. At. Mol. Phys. 11, 1 (1975).

${ }^{6}$ R. E. Olson, J. Phys. B 13, 483 (1980).

${ }^{7}$ H. A. Bethe and E. A. Salpeter, Quantum Mechanics of One-and Two-Electron Atoms (Academic, New York, 1957).

${ }^{8}$ T. F. Gallagher, L. M. Humphrey, W. E. Cooke, R. M. Hill, and S. A. Edelstein, Phys. Rev. A 16, 1098 (1977).

${ }^{9}$ T. F. Gallagher and W. E. Cooke, Appl. Phys. Lett. 34, 369 (1979).

${ }^{10}$ T. W. Ducas, W. P. Spencer, A. G. Vaidyanathan, W. H. Hamilton, and D. Kleppner, Appl. Phys. Lett. 35, 382 (1979).

${ }^{11}$ H. Figger, G. Leuchs, R. Straubinger, and H. Walther, Opt. Commun. 33,37 (1980).

${ }^{12}$ R. E. Olson and A. Salop, Phys. Rev. A 16,531 (1977).

${ }^{13}$ D. Banks and J. G. Leopold, J. Phys. B 11, 37, L5, 2833 (1978).

${ }^{14}$ J. G. Lodge, I. C. Percival, and D. Richards, J. Phys. B $\underline{9}, 239$ (1976).

\title{
Coherent Compton Effect
}

\author{
J. A. Golovchenko, D. R. Kaplan, (a) B. Kincaid, R. Levesque, \\ A. Meixner, M. F. Robbins, and J. Felsteiner ${ }^{(b)}$ \\ Bell Laboratories, Murray Hill, New Jersey 07974 \\ (Received 23 February 1981)
}

\begin{abstract}
Interference effects have been observed when coherently related $\mathrm{x}$-ray beams are Compton scattered from an atomic system. We use dynamical diffraction methods to prepare the initial $\mathrm{x}$-ray state and observe the coherent Compton effect in both crosssection and profile measurements.
\end{abstract}

PACS numbers: $32.90 .+\mathrm{a}, 32.30 . \mathrm{Rj}$

The conventional method of observing the Compton effect involves the preparation of an $\mathrm{x}$-ray photon state of reasonably sharp momentum which is subsequently scattered from an electronic system. The final state of the total system consists of a recoiling electron and a scattered photon of reduced energy. If the target electron is initially at rest or in a state of uniform motion a unique relationship between photon scattering angle and energy loss results from simple kinematic considerations. However, if the electron is not free, but bound in an atom or solid, a range of photon energy losses will belong to a particular scattering angle. This range reflects the momentum spread in the bound-electron state. The total spectrum from all ground-state electrons in the target is commonly referred to as a Compton profile. Interest in this subject has continued into recent years precisely because electronic momentum distribution functions for atomic and solidstate systems can be extracted from Compton profiles. ${ }^{1}$

In the following we report on an extension of these notions to the case where the incident photon is no longer in a momentum eigenstate, but must be represented by a more complex coherent superposition of momentum eigenstates. From the general principle of superposition of quantum amplitudes we may anticipate the scattered photon intensity to be the sum of intensities expected from individual experiments for each momentum component in the incident state plus interference 
effects arising in those final states that can be reached from any of the incident component states.

Consider the following concrete example. It corresponds closely to an experimental realization to be discussed shortly. The photon is prepared in a superposition of two coherently related momentum eigenstates differing in direction but not magnitude of momentum. This "beam" is scattered off an electron into an energy dispersive detector looking at a fixed angle relative to the target. If the target electron is initially at rest (and free) our Compton profile will consist of two sharp peaks at different energies, one for each scattering angle from the incident beam (which contains two directions). The result is just the sum of the experimental results expected for each component of the incident beam. There is no interference; the experimenter can easily determine from which component of the incident beam the scattering took place.

Imagine, on the other hand, scattering from an electron bound to an atom. The sharp peaks broaden, reflecting the spread in momentum in the electron state. If we let the binding increase the peaks will continually broaden until they significantly overlap. A count occurring in this overlap region can result from either of the incident photon components. Examination of the recoilelectron final state does not completely resolve the uncertainty for there will also be two peaks in the electron recoil directions that increasingly overlap with increased binding, and again the peak to which an overlap event belongs will be uncertain. It is therefore from the most deeply bound states that we expect the strongest interference effects. In addition the results of experiments with bound electrons will depend on the actual spatial position of the atom, since the initial photon state is not translationally invariant. In fact atoms interacting with this state are equivalent only when their position along the direction of photon momentum difference, $\Delta \overrightarrow{\mathrm{P}}$, is $2 \pi \hbar /|\Delta P|$. Averaging over atom positions along this direction will "smear out" all expected interference effects.

To observe the coherent Compton effect one must (1) prepare an X-ray photon state with coherently related momentum components, (2) place target atoms in equivalent positions in the $\mathrm{x}$-ray field, and (3) measure total Compton yields and/ or profiles at some angle and show that these depend upon the phase between photon components in the incident state.

These conditions are met in an experiment that observes the Compton scattering from a crystal in which the $\mathrm{x}$-ray beam is being strongly Bragg diffracted. In the near-surface region of the crystal the incident and Bragg-diffracted beams overlap in space, are coherently related, differ in momentum by a reciprocal lattice vector, and can be of similar strengths. Indeed the lattice atoms are in exactly equivalent positions in the periodic field intensity since they are responsible for it! The relative phases of the two-component $\mathrm{x}$-ray field can even be varied experimentally. The utility of these features of this $x$-ray wave field is by now well established in fluorescent scattering for impurity atom location ${ }^{2}$ and we take the quantitative success of this enterprise as a measure of our ability to prepare the well-defined photon states we require.

All the essential features of the x-ray field in the crystal can be deduced from the dynamical theory of $\mathrm{x}$-ray diffraction. ${ }^{3}$ It predicts that for large perfect crystals an angular (band-gap) range exists within which an external $x$-ray beam will be essentially totally reflected from the crystal. The internal evanescent wave field in this region is of the standing-wave type with a periodicity of the Miller planes responsible for the diffraction. The phase of the diffracted wave (and therefore of the standing wave) changes by $\pi$ in changing angle from one side of the totally reflecting region to the other. In addition there is an envelope of exponential extinction of the beam with increasing depth into the sample that depends strongly on angular orientation.

We have observed the coherent Compton effect in measurements of total Compton yields and Compton profiles. Figure 1 contains a small sketch of a total-yield experiment. A Mo $K \alpha \mathrm{x}-$ ray beam from a $1-\mathrm{kW}$-ray tube was collimated with an asymmetric (220) silicon collimator crystal to an effective angular divergence of one-seventh the (220) natural reflection width. This beam was then directed onto a symmetrically cut (220) silicon crystal and both the elastically (Bragg-) reflected intensity and inelastic Compton scattering were observed as a function of angular orientation of this crystal in the vicintiy of the Bragg condition $\left(\theta_{\text {Bragg }}=10.6^{\circ}\right)$. The reflected beam was monitored with a NaI scintillation detector and the Compton yield was observed normal to the crystal surface with a $\mathrm{Si}(\mathrm{Li})$ detector that could easily resolve the Compton and thermal diffuse scattering peaks. The method of controlling and stabilizing the crystal angle has been described elsewhere. ${ }^{4}$ 


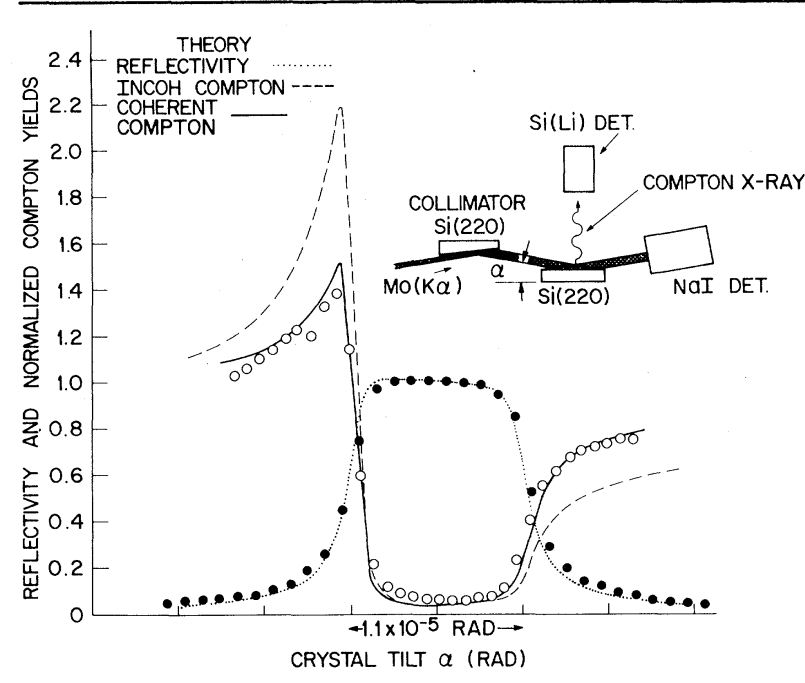

FIG. 1. Angular-yield data showing reflecivity and Compton-effect results.

The closed circles in Fig. 1 show the experimental angular-reflectivity results. The angular band gap is clearly seen and it agrees well with the dynamical-theory prediction (dotted curve) which is folded with the angular-resolution function of the collimator crystal. The open circles show the angular dependence of the Compton yield in the $\mathrm{Si}(\mathrm{Li})$ detector. These results have been normalized to the yield obtained in the absence of diffraction. Basically we observe a large dip in the total reflectivity angular region with strongly asymmetric wings to the left and right. The gross structure is reproduced by a calculation involving no coherency effects in the Compton scattering (dashed curve). That is, the dynamical theory is used to predict the intensity and penetration depth of both incident and reflected beams and the total Compton yield is obtained as the independent sum of the yields from the two beams. The strong central dip is due to the effective sample thickness decrease resulting from extinction. As one proceeds in angle from the central region towards the corners of the reflectivity curve the extinction length becomes infinite in principle and the effective sample thickness contributing to the Compton yield becomes limited by photoelectric absorption. The absorption coefficients are, however, quite different at the two sides of the reflectivity curve due to the standing-wave fields. On the left-hand side the standing-wave nodes are on the (220) atomic planes and consequently absorption is low and effective sample thickness is quite large. On the right-hand side the antinodes are on the planes and absorption is anomalously high and effective sample thickness is considerably reduced. (Calculations similar to this one where one does not consider interference in the Compton channel have been presented by Annaka, Kikuta, and Kohra. ${ }^{5}$ )

All of this explains the dip and basic asymmetry in the data; however, good quantitative agreement is clearly not obtained. The solid curve in Fig. 1 contains all the effects of the dashed curve, but the interference effects discussed in the introduction of this paper have also been included. The asymmetry is greatly reduced and agreement with the data is clearly enhanced.

Why is the asymmetry so strongly reduced? While a proper discussion requires a more detailed momentum-space approach, we can see heuristically that when the standing-wave solutions have nodes on the atomic planes, core-electron contributions should be eliminated from the total yield. In line with the discussion in the introduction we note that high-momentum components associated with atomic cores are most susceptible to interference effects due to overlap in the two Compton profiles and electron recoil distributions. This explains the reduction from the dashed to solid curve on the left-hand side of the figure. The analogous increase in core contribution for standing waves on the planes explains the increase on the right-hand side.

As a further demonstration of the coherent Compton effect that is somewhat less dependent on the details of the dynamical theory for its interpretation, we show Compton profiles obtained when the standing-wave antinodes are between the diffracting atomic planes $(A)$ and on them $(B)$. The two spectra are shown in Fig. 2 with the geometry of the experiment in the inset.

A beam of $x$ rays from the CHESS (Cornell High Energy Synchrotron Source) synchrotron facility was intercepted by a recently designed $x$-ray monochromator ${ }^{6}$ containing an asymmetric crystal for beam collimation similar to that previously described. The resulting $17.2-\mathrm{keV} \mathrm{x}$-ray beam fell on a symmetrically cut (220) silicon crystal set to the (220) diffraction condition. Use of the synchrotron source resulted in beam on sample improvements of $\sim 10^{2}$ compared with a $1-\mathrm{kW}$ finefocus $x$-ray tube. The crystal was scanned in angle to observe a reflectivity curve similar to that shown in Fig. 1. In this experiment the $\mathrm{Si}(\mathrm{Li}) \mathrm{de}-$ tector was set to observe Compton-scattered photons at $150^{\circ}$ to both incident and diffracted beams. Detector resolution as determined from the quasielastic thermal-diffuse-scattering peaks in Fig. 


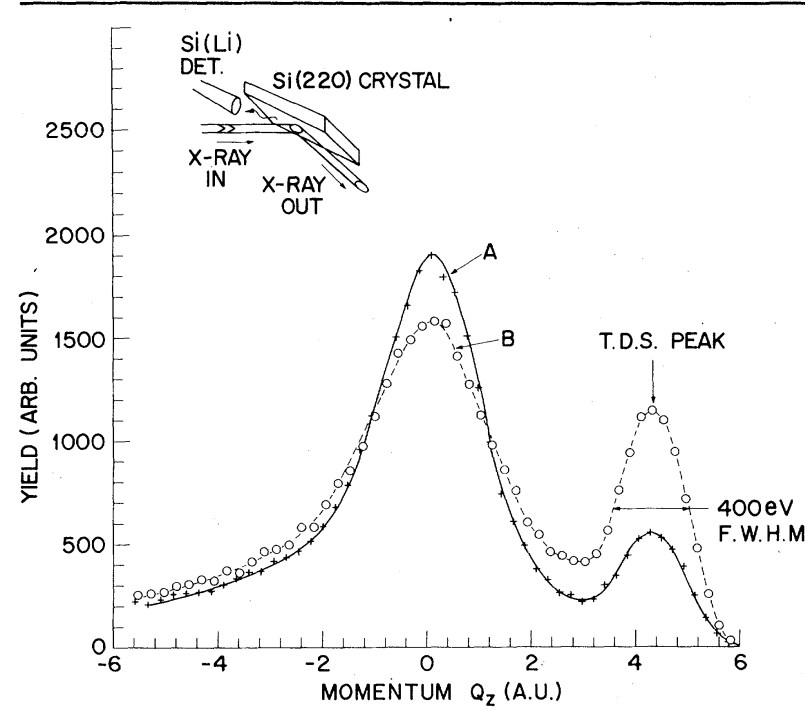

FIG. 2. Compton profiles: Curve $A$, antinodes between planes; curve $B$, antinodes on planes.

2 was $400 \mathrm{eV}$ full width at half maximum. The detector energy scale has been changed to an electron projected momentum scale and the areas under the Compton profiles of the two spectra have been set equal.

A clear difference in shape between the two curves is evident. When the standing-wave antinodes are between the planes (curve $A$ ) interference effects have reduced the broad core contributions and a more sharply peaked momentum profile is observed. On the other hand, curve $B$ with standing waves on the planes is broader because of increased core contribution from the interference. We note that approximately $20 \%$ of the yield in both curves of Fig. 2 is a background of scattering from the diffracted beam alone after leaving the crystal. Since reflected beam intensities were equal in both experiments, so was the background. Also the spectral effects of multiple scattering in the sample were estimated theoretically by a method similar to that of Felsteiner and Pattison ${ }^{7}$ and were found to be negligible. At any rate, the only difference in the experiments from which these curves resulted is that the phase of the diffracted beam is altered by $\pi$ and consequently the difference in spectral shape is a clear manifestation of interference in the co- herent Compton effect.

In conclusion, we have observed strong interference effects in Compton scattering from coherently related incident states both in total cross-section and profile measurements. Both the momentum-space and coordinate-space arguments mentioned in this paper suggest that these effects can be used to probe the specific contributions to a total Compton profile. In particular, core effects can be suppressed to more clearly isolate momentum components associated with "outer"-electron solid-state effects. Clearly higher energy resolution in the Compton profiles is possible and is now required to obtain useful results in this area.

We would like to acknowledge useful discussions and encouragement from W. L. Brown and Phil Platzman of Bell Laboratories. In addition, we would like to thank Professor Batterman for cooperation in our utilization of the CHESS facility at Cornell University.

\footnotetext{
(a)Also at Princeton University, Princeton, N. J. 08540 .

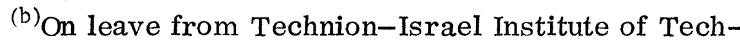
nology, Haifa, Israel.

${ }^{1}$ Compton Scattering, edited by Brian Williams (McGraw-Hill, New York, 1977).

${ }^{2}$ B. W. Batterman, Phys. Rev. 133, A759 (1964), and Phys. Rev. Lett. 22, 703 (1969); J. A. Golovchenko, B. W. Batterman, and W. L. Brown, Phys. Rev. B 10, 4239 (1974); S. K. Anderson, J. A. Golovchenko, and G. Mair, Phys. Rev. Lett. 37, 1141 (1976); M. V. Kruglov, V. N. Shchemeley, and G. G. Kareva, Phys. Status Solidi (a) 46, 343 (1978); P. L. Cowan, J. A. Golovchenko, and M. F. Robbins, Phys. Rev. Lett. 44, 1680 (1980).

${ }^{3} \mathrm{R}$. W. James, The Optical Principles of the Diffraction of X-Rays (Bell, London, 1950).

${ }^{4}$ G. L. Miller, R. Boie, P. L. Cowan, J. A. Golovchenko, R. Kerr, and D. Robinson, Rev. Sci. Instrum. 50,8 (1979); S. K. Andersen, P. K. Bhattacharya, J. Golovchenko, N. Hertel, and G. Mair, J. Phys. E 12, 1063 (1979).

${ }^{5}$ S. Annaka, S. Kikuta, and K. Kohra, J. Phys. Soc. Jpn. 21, 1559 (1966)。

${ }^{6}$ J. A. Golovchenko, R. A. Levesque, and P. L. Cowan, to be published.

${ }^{7}$ J. Felsteiner and P. Pattison, Nucl. Instrum. Methods 124,449 (1975).
} 